九州大学学術情報リポジトリ

Kyushu University Institutional Repository

\title{
The Recurrence of Dynamic Fuzzy Systems
}

Yoshida, Yuji

Faculty of Economics, Kitakyushu University

http://hdl. handle. net/2324/3179

出版情報 : RIFIS Technical Report. 80，1994-02-10. Research Institute of Fundamental Information Science, Kyushu University バージョン:

権利関係 : 


\section{RIFIS Technical Report}

The Recurrence of Dynamic Fuzzy Systems

Yuji Yoshida

February 10,1994

Research Institute of Fundamental Information Science

Kyushu University 33

Fukuoka 812, Japan

E-mail: yoshida@rifis.sci.kyushu-u.ac.jp Phone:092-641-1101 ex.4484 


\title{
THE RECURRENCE OF DYNAMIC FUZZY SYSTEMS
}

\author{
Yuji YOSHIDA \\ Faculty of Economics, Kitakyushu University, \\ Kitagata, Kokuraminami, Kitakyushu 802, Japan
}

\begin{abstract}
This paper analyses a recurrent behavior of dynamic fuzzy systems defined by fuzzy relations on a Euclidean space. By introducing a recurrence for crisp sets, we prove probability-theoretical properties for the fuzzy systems. In the contractive case in [5], the existence of the maximum recurrent set is proved. As another case, we introduce a monotonicity for fuzzy relations, which is extended from the linear structure in [11]. In the monotone case we prove the existence of the arcwise connected maximal recurrent sets.
\end{abstract}

Keyword : Recurrence; dynamic fuzzy systems; fuzzy relations; contraction; monotonicity; superharmonic property.

\section{Introduction and notations}

Limit theorems of a sequence of fuzzy sets defined successively by fuzzy relations are first studied by Bellman and Zadeh [1]. They considered a sequence of fuzzy numbers in a finite space and solved a fuzzy relational equation written in matrix form. Kurano et al. [5] and Yoshida et al. [11], under a contractive condition, studied the limiting behavior of fuzzy sets defined by the dynamic fuzzy system with a compact space. We, in [5], proved the existence and uniqueness of the solution for the fuzzy relational equation, and in [11], developed, under a linear structure, a potential theory of fuzzy relations on the positive orthant of a Euclidean space.

Our objective is to study maximal recurrence of the dynamic fuzzy systems defined by fuzzy relations on a Euclidean space. We introduce, for every level $\alpha \in(0,1]$, a recurrence for crisp sets and we call it $\alpha$-recurrence. In Section 2 we prove, on the $\alpha$-recurrent crisp sets, various probability-theoretical properties in the class of fuzzy sets satisfying a fuzzy relational inequality, which is a generalization of the fuzzy relational equations in [5] and which is also satisfied by optimal fuzzy goals in fuzzy dynamic programming of [1], [2], [10]. Further we establish the balayage theorem, which is well-known regarding Markov chains, for the dynamic fuzzy system. In Section 3 we introduce $\alpha$-recurrence and represent the union of all $\alpha$-recurrent sets by the fuzzy relation. In Section 4 we deal with the contractive case in [5]. We give an explicit solution of the fuzzy relational equation in [5] and we prove that the $\alpha$-cut of the solution is the maximum $\alpha$-recurrent set. In Section 5 we introduce a certain monotonicity for the fuzzy relation, which is a natural extension of one-dimensional fuzzy relations with the linear structure in [11]. Then we prove that at most countable maximal $\alpha$-recurrent sets exist and that each maximal $\alpha$-recurrent set is arcwise connected. In Section 6 numerical examples are given to illustrate our idea. 
In the remainder of this section, we describe the notations for dynamic fuzzy systems defined by fuzzy relations on finite-dimensional Euclidean spaces and give some fundamental results for stopping times from Yoshida [10].

Let $S$ be a metric space. We write a fuzzy set on $S$ by its membership function $\tilde{s}: S \mapsto[0,1]$ and an ordinary set $A(\subset S)$ by its indicator function $1_{A}: S \mapsto\{0,1\}$. The $\alpha$-cut $\tilde{s}_{\alpha}$ is defined by

$$
\tilde{s}_{\alpha}:=\{x \in S \mid \tilde{s}(x) \geq \alpha\}(\alpha \in(0,1]) \quad \text { and } \quad \tilde{s}_{0}:=\operatorname{cl}\{x \in S \mid \tilde{s}(x)>0\},
$$

where cl denotes the closure of a set. $\mathcal{F}(S)$ denotes the set of all fuzzy sets $\tilde{s}$ on $S$ satisfying the following conditions (F.i) and (F.ii) :

(F.i) $\tilde{s}_{\alpha} \in \mathcal{E}(S)$ for $\alpha \in[0,1]$;

(F.ii) $\bigcap_{\alpha^{\prime}<\alpha} \tilde{s}_{\alpha^{\prime}}=\tilde{s}_{\alpha} \quad$ for $\alpha \in(0,1]$,

where

$$
\mathcal{E}(S):=\left\{A \mid A=\bigcup_{n=0}^{\infty} C_{n}, C_{n} \text { are closed subsets of } S(n=0,1,2, \cdots)\right\} .
$$

We also define

$$
\mathcal{G}(S):=\left\{\text { fuzzy sets } \tilde{s} \text { on } S \mid \text { there exists }\left\{\tilde{s}_{n}\right\}_{n \in \mathrm{N}} \subset \mathcal{F}(S) \text { satisfying } \tilde{s}=\bigvee_{n \in \mathrm{N}} \tilde{s}_{n}\right\},
$$

where $\mathbf{N}:=\{0,1,2,3, \cdots\}$ and for a sequence of fuzzy sets $\left\{\tilde{s}_{n}\right\}_{n \in \mathbf{N}}$ on $S$ we define

$$
\bigwedge_{n \in \mathrm{N}} \tilde{s}_{n}(x):=\inf _{n \in \mathrm{N}} \tilde{s}_{n}(x) \text { and } \bigvee_{n \in \mathrm{N}} \tilde{s}_{n}(x):=\sup _{n \in \mathrm{N}} \tilde{s}_{n}(x) \quad x \in S
$$

Let a time space by $\mathbf{N}$ and put $\overline{\mathbf{N}}:=\mathrm{N} \cup\{\infty\}$. Let a state space $E$ be a finite-dimensional Euclidean space. We put a path space by $\Omega:=\prod_{k=0}^{\infty} E$ and we write a sample path by $\omega=(\omega(0), \omega(1), \omega(2), \cdots) \in \Omega$. We define a map $X_{n}(\omega):=\omega(n)$ and a shift $\theta_{n}(\omega):=$ $(\omega(n), \omega(n+1), \omega(n+2), \cdots)$ for $n \in \mathbf{N}$ and $\omega=(\omega(0), \omega(1), \omega(2), \cdots) \in \Omega$. We put $\sigma$-fields by $\mathcal{M}_{n}:=\sigma\left(X_{0}, X_{1}, \cdots, X_{n}\right)^{1}$ for $n \in \mathbf{N}$ and $\mathcal{M}:=\sigma\left(\bigcup_{n \in \mathrm{N}} \mathcal{M}_{n}\right)^{2}$. Let $\Delta$ be not a point of $E$ and put $E_{\Delta}:=E \cup\{\Delta\}$. We can extend the state space $E$ to $E_{\Delta}$, setting $\tilde{s}(\Delta):=0$ for $\tilde{s} \in \mathcal{G}\left(E_{\Delta}\right)$ and $X_{\infty}(\omega):=\Delta$ for $\omega \in \Omega([10$, Section 2$])$. Let $\tilde{q}$ be an upper semi-continuous binary relation on $E \times E$ satisfying the following normality condition :

$$
\sup _{x \in E} \tilde{q}(x, y)=1(y \in E) \text { and } \sup _{y \in E} \tilde{q}(x, y)=1(x \in E) .
$$

We call $\tilde{q}$ a fuzzy relation. We define a fuzzy expectation : For an initial state $x \in E$ and an $\mathcal{M}$-measurable fuzzy set $h \in \mathcal{F}(\Omega)$,

$$
E_{x}(h):=f_{\{\omega \in \Omega: \omega(0)=x\}} h(\omega) \mathrm{d} \tilde{P}(\omega),
$$

\footnotetext{
${ }^{1}$ It denotes the smallest $\sigma$-field on $\Omega$ relative to which $X_{0}, X_{1}, \cdots, X_{n}$ are measurable.

${ }^{2}$ It denotes the smallest $\sigma$-field generated by $\bigcup_{n \in \mathbb{N}} \mathcal{M}_{n}$.
} 
where $\tilde{P}$ is the following possibility measure :

$$
\tilde{P}(\Lambda):=\sup _{\omega \in \Lambda} \bigwedge_{n \in \mathrm{N}} \tilde{q}\left(X_{n} \omega, X_{n+1} \omega\right) \quad \Lambda \in \mathcal{M}
$$

and $f \mathrm{~d} \tilde{P}$ denotes Sugeno integral (Sugeno [9]). Then the fuzzy expectation has the following property.

Lemma 1.1 ([10, Section 3]). For an $\mathcal{M}$-measurable sequence $\left\{h_{n}\right\}_{n \in \mathbf{N}} \subset \mathcal{G}(\Omega)$, it holds that

$$
\bigvee_{n \in \mathrm{N}} E_{x}\left(h_{n}\right)=E_{x}\left(\bigvee_{n \in \mathrm{N}} h_{n}\right) \quad x \in E
$$

We need the first entry times (the first hitting times) of a set, which is adapted to the dynamic fuzzy system $X:=\left\{X_{n}\right\}_{n \in \mathrm{N}}$, in order to define a recurrence of sets in Section 3. We define

$$
\mathcal{E}:=\{A \mid A \in \mathcal{E}(E) \text { and } E \backslash A \in \mathcal{E}(E)\}
$$

and we call a map $\tau: \Omega \mapsto \overline{\mathbf{N}}$ an $\mathcal{E}$-stopping time if

$$
\{\tau=n\} \in \mathcal{M}_{n} \cap \mathcal{E}(\Omega) \quad n \in \mathbf{N}
$$

For example, a constant stopping time i.e. $\tau=n_{0}$ for some $n_{0} \in \mathbf{N}$, is an $\mathcal{E}$-stopping time. For $A \in \mathcal{E}$ we put

$$
\begin{gathered}
\tau_{A}(\omega):=\inf \left\{n \in \mathbf{N} \mid X_{n}(\omega) \in A\right\} \quad \omega \in \Omega ; \\
\sigma_{A}(\omega):=\inf \left\{n \in \mathbf{N} \mid n \geq 1, X_{n}(\omega) \in A\right\} \quad \omega \in \Omega,
\end{gathered}
$$

where the infimums of the empty set are understood to be $+\infty$. Then the first entry time $\tau_{A}$ of $A$ and the first hitting time $\sigma_{A}$ of $A$ are also $\mathcal{E}$-stopping times ([10, Lemma 1.5]).

Define a map $P: \mathcal{G}(E) \mapsto \mathcal{G}(E)$ by

$$
P \tilde{s}(x):=E_{x}\left(\tilde{s}\left(X_{1}\right)\right)=\sup _{y \in E}\{\tilde{q}(x, y) \wedge \tilde{s}(y)\} \quad x \in E \quad \text { for } \tilde{s} \in \mathcal{G}(E)
$$

where we write binary operations $a \wedge b:=\min \{a, b\}$ and $a \vee b:=\max \{a, b\}$ for $a, b \in[0,1]$. We call $P$ a fuzzy transition defined by the fuzzy relation $\tilde{q}$. We also define $n$-steps fuzzy transitions $P_{n}: \mathcal{G}(E) \mapsto \mathcal{G}(E), n \in \mathbf{N}$, by

$$
P_{n} \tilde{s}:=E \cdot\left(\tilde{s}\left(X_{n}\right)\right)=\sup _{y \in E}\left\{\tilde{q}^{n}(\cdot, y) \wedge \tilde{s}(y)\right\} \quad \text { for } \tilde{s} \in \mathcal{G}(E),
$$

where for $n \in \mathbf{N}$

$$
\tilde{q}^{1}(x, y):=\tilde{q}(x, y) \text { and } \tilde{q}^{n+1}(x, y):=\sup _{z \in E}\left\{\tilde{q}^{n}(x, z) \wedge \tilde{q}(z, y)\right\} \quad x, y \in E .
$$


Further for an $\mathcal{E}$-stopping time $\tau$, a fuzzy transition $P_{\tau}: \mathcal{G}(E) \mapsto \mathcal{G}(E)$ is defined by

$$
P_{\tau} \tilde{s}:=E \cdot\left(\tilde{s}\left(X_{\tau}\right)\right) \text { for } \tilde{s} \in \mathcal{G}(E),
$$

where $X_{\tau}:=X_{n}$ on $\{\tau=n\}, n \in \overline{\mathbf{N}}$.

The fuzzy transition $\left\{P_{n}\right\}_{n \in N}$ has the following property :

$$
P_{0}=I \text { (identity), } \quad P_{1}=P \quad \text { and } \quad P_{m+n}=P_{m} P_{n}(m, n \in \mathbf{N}) .
$$

Further it also has a semi-group property with respect to $\mathcal{E}$-stopping times.

Lemma 1.2 ([10, Corollary 2.1]). It holds that

$$
P_{\sigma} P_{\tau}=P_{\sigma+\tau \circ \theta_{\sigma}} \text { on } \mathcal{G}(E) \text { for finite } \mathcal{E} \text {-stopping times } \sigma \text { and } \tau \text {. }
$$

\section{Transitive closures and $P$-superharmonic fuzzy sets}

We define a partial order $\geq$ on $\mathcal{G}(E):$ For $\tilde{s}, \tilde{r} \in \mathcal{G}(E)$

$$
\tilde{s} \geq \tilde{r} \Longleftrightarrow \tilde{s}(x) \geq \tilde{r}(x) \quad x \in E .
$$

Definition $([10$, Section 4$])$. A fuzzy set $\tilde{s}(\in \mathcal{G}(E))$ is called $P$-harmonic ( $P$-superharmonic) provided that

$$
\tilde{s}=P \tilde{s} \quad(\tilde{s} \geq P \tilde{s} \quad \text { resp. }) .
$$

Clearly a constant fuzzy set, $\tilde{s}=\beta$ for some $\beta \in[0,1]$, is $P$-superharmonic. We represent the fuzzy set by $\beta$ simply.

In this section we investigate $P$-superharmonic property regarding fuzzy sets and we show the balayage theorem for $P$-superharmonic fuzzy sets. Using the results, we give a simple characterization for hitting possibilities of a set $A(\in \mathcal{E})$ by transitive closures. First we prove preliminary lemmas for $P$-superharmonic fuzzy sets, which are well-known property in the classical probability theory $([8])$.

\section{Lemma 2.1 .}

(i) If $\tilde{s}_{1}$ and $\tilde{s}_{2}$ are $P$-superharmonic, then $\tilde{s}_{1} \wedge \tilde{s}_{2}$ is also $P$-superharmonic.

(ii) If $\left\{\tilde{s}_{n}\right\}_{n \in \mathrm{N}}$ is a sequence of $P$-harmonic ( $P$-superharmonic) fuzzy sets, then $\bigvee_{n \in \mathrm{N}} \tilde{s}_{n}$ is also $P$-harmonic ( $P$-superharmonic resp.).

Proof. (i) We can easily check $\tilde{s}_{1} \wedge \tilde{s}_{2} \in \mathcal{G}(E)$, using [10, Lemma 1.1]. Since the fuzzy transition $P$ preserves the order $\geq$ on $\mathcal{G}(E)$, we have

$$
\tilde{s}_{1} \geq P \tilde{s}_{1} \geq P\left(\tilde{s}_{1} \wedge \tilde{s}_{2}\right) \quad \text { and } \quad \tilde{s}_{2} \geq P \tilde{s}_{2} \geq P\left(\tilde{s}_{1} \wedge \tilde{s}_{2}\right)
$$


Therefore $\tilde{s}_{1} \wedge \tilde{s}_{2}$ is $P$-superharmonic.

(ii) It is trivial that $\bigvee_{n \in \mathrm{N}} \tilde{s}_{n} \in \mathcal{G}(E)$. By Lemma 1.1,

$$
\bigvee_{n \in \mathrm{N}} \tilde{s}_{n} \geq \bigvee_{n \in \mathrm{N}} P \tilde{s}_{n}=P\left(\bigvee_{n \in \mathrm{N}} \tilde{s}_{n}\right)
$$

Therefore $\bigvee_{n \in \mathbb{N}} \tilde{s}_{n}$ is $P$-superharmonic. The $P$-harmonic case is similar.

\section{Lemma 2.2.}

(i) If $\tilde{s}$ is $P$-superharmonic, then

$$
P_{\sigma} \tilde{s} \geq P_{\tau} \tilde{s} \quad \text { for all } \mathcal{E} \text {-stopping times } \sigma \text { and } \tau \text { such that } \sigma \leq \tau \text {. }
$$

(ii) If $\tilde{s}$ is $P$-harmonic, then

$$
P_{\sigma} \tilde{s}=P_{\tau} \tilde{s} \quad \text { for all } \mathcal{E} \text {-stopping times } \sigma \text { and } \tau \text { such that } \sigma \leq \tau<\infty \text {. }
$$

Proof. (i) We check this lemma along the proof of [8, Proposition II-1.9]. Let $\sigma$ and $\tau$ be $\mathcal{E}$-stopping times such that $\sigma \leq \tau \leq \sigma+1$. Let $\Lambda_{n}:=\{\sigma=n\} \cap\{\tau=n+1\} \in \mathcal{M}_{n}$ and $\Gamma_{n}:=\{\sigma=\tau=n\} \in \mathcal{M}_{n}$ for $n \in \mathbf{N}$. By [10, Theorem 2.1], for $n \in \mathbf{N}$

$$
E_{x}\left(\tilde{s}\left(X_{\sigma}\right) \wedge 1_{\Lambda_{n}}\right) \geq E_{x}\left(P \tilde{s}\left(X_{n}\right) \wedge 1_{\Lambda_{n}}\right)=E_{x}\left(\tilde{s}\left(X_{n+1}\right) \wedge 1_{\Lambda_{n}}\right)=E_{x}\left(\tilde{s}\left(X_{\tau}\right) \wedge 1_{\Lambda_{n}}\right) \quad x \in E .
$$

Using Lemma 1.1, we obtain

$$
\begin{aligned}
P_{\sigma} \tilde{s}(x) & =\bigvee_{n \in \mathrm{N}}\left(E_{x}\left(\tilde{s}\left(X_{\sigma}\right) \wedge 1_{\Lambda_{n}}\right) \vee E_{x}\left(\tilde{s}\left(X_{\sigma}\right) \wedge 1_{\Gamma_{n}}\right)\right) \\
& \geq \bigvee_{n \in \mathbb{N}}\left(E_{x}\left(\tilde{s}\left(X_{\tau}\right) \wedge 1_{\Lambda_{n}}\right) \vee E_{x}\left(\tilde{s}\left(X_{\tau}\right) \wedge 1_{\Gamma_{n}}\right)\right) \\
& =P_{\tau} \tilde{s}(x) \quad x \in E .
\end{aligned}
$$

More generally, for $\mathcal{E}$-stopping times $\sigma$ and $\tau$ such that $\sigma \leq \tau$,

$$
P_{\sigma} \tilde{s} \geq P_{(\sigma+1) \wedge \tau} \tilde{s} \geq \cdots \geq P_{(\sigma+n) \wedge \tau} \tilde{s} \geq \cdots \quad \text { for } n \in \mathrm{N} .
$$

Here, from [10, Lemma 1.1(i)], we have the following facts :

$$
\begin{gathered}
\{\tau \leq \sigma+n<\infty\}=\bigcup_{l, m \in \mathrm{N}: l \leq m+n}(\{\tau=l\} \cap\{\sigma=m\}) \in \mathcal{E}(\Omega) ; \\
\{\tau<\infty\}=\bigcup_{l \in \mathrm{N}}\{\tau=l\} \in \mathcal{E}(\Omega) ; \\
\bigcup_{n \in \mathbb{N}}\{\tau \leq \sigma+n<\infty\}=\{\tau<\infty\} .
\end{gathered}
$$

By Lemma 1.1 and (2.1),

$$
P_{\sigma} \tilde{s}(x) \geq \bigvee_{n \in \mathrm{N}} P_{(\sigma+n) \wedge \tau} \tilde{s}(x) \geq \bigvee_{n \in \mathrm{N}} E_{x}\left(\tilde{s}\left(X_{\tau}\right) \wedge 1_{\{\tau \leq \sigma+n<\infty\}}\right)=E_{x}\left(\tilde{s}\left(X_{\tau}\right) \wedge 1_{\{\tau<\infty\}}\right)=P_{\tau} \tilde{s}(x)
$$


for $x \in E$. Therefore we get (i). We can check (ii) similarly.

We show the balayage theorem for the dynamic fuzzy system $X$. The theorem plays one of important roles to analyse recurrence for the fuzzy relation $\tilde{q}$ in Section 3.

Theorem 2.1. Let $\tilde{s}$ be $P$-superharmonic and let a set $A \in \mathcal{E}$. Then $P_{\tau_{A}} \tilde{s}$ is the smallest $P$-superharmonic fuzzy set which dominates $\tilde{s} \wedge 1_{A}$.

Proof. We check this theorem along the proof of [8, Theorem II-2.1] for the classical Markov chain. It is trivial that $P_{\tau_{A}} \tilde{s}=\tilde{s}$ on $A . P_{\tau_{A}} \tilde{s}$ is $P$-superharmonic since $P P_{\tau_{A}} \tilde{s}=$ $P_{\sigma_{A}} \tilde{s} \leq P_{\tau_{A}} \tilde{s}$ by Lemmas 1.2 and 2.2(i). Therefore $P_{\tau_{A}} \tilde{s}$ is $P$-superharmonic and dominates $\tilde{s} \wedge 1_{A}$. Further let $\tilde{r}$ be $P$-superharmonic such that $\tilde{r} \geq \tilde{s} \wedge 1_{A}$. Then

$$
\tilde{r}(x) \geq P_{\tau_{A}} \tilde{r}(x)=E_{x}\left(\tilde{r}\left(X_{\tau_{A}}\right) \wedge 1_{\left\{\tau_{A}<\infty\right\}}\right) \geq E_{x}\left(\tilde{s}\left(X_{\tau_{A}}\right) \wedge 1_{\left\{\tau_{A}<\infty\right\}}\right)=P_{\tau_{A}} \tilde{s}(x) \quad x \in E .
$$

Thus $P_{\tau_{A}} \tilde{s}$ has the desired property and so we get this theorem.

We define an operator $G:=\bigvee_{n \in \mathrm{N}} P_{n}$ on $\mathcal{G}(E)$. Then we note that

$$
P G 1_{\{y\}}(x)=\bigvee_{n \geq 1} P_{n} 1_{\{y\}}(x)=\sup _{n \geq 1} \tilde{q}^{n}(x, y) \quad x, y \in E .
$$

This is called a transitive closure $([3$, Section 3.3$])$. In this paper we also call $P G$ a transitive closure. Now we need to investigate the operator $G$ in order to analyse the transitive closure $P G:=\bigvee_{n \geq 1} P_{n}$. We have the following properties regarding $G$.

Lemma 2.3 ([10, Lemma 4.1(ii)]). Let $\tilde{s} \in \mathcal{G}(E)$. Then :

(i) It holds that

$$
G \tilde{s}=\tilde{s} \vee P(G \tilde{s})
$$

(ii) $G \tilde{s}$ is the smallest $P$-superharmonic dominating $\tilde{s}$.

Lemma 2.4. Let $\tilde{s} \in \mathcal{G}(E)$. Then $\tilde{s}$ is $P$-superharmonic if and only if

$$
\tilde{s}=G \tilde{s}
$$

Proof. Let $\tilde{s}$ be $P$-superharmonic. Then

$$
\tilde{s}=\tilde{s} \vee P \tilde{s} \vee P_{2} \tilde{s} \vee \cdots \vee P_{n} \tilde{s} \quad \text { for all } n \in \mathbf{N} .
$$

So we obtain (2.2). The converse proof is trivial.

For $A \in \mathcal{E}(E)$ we introduce an operator $I_{A}: \mathcal{G}(E) \mapsto \mathcal{G}(E)$ by

$$
I_{A} \tilde{s}:=\tilde{s} \wedge 1_{A} \quad \tilde{s} \in \mathcal{G}(E) .
$$


We define a sequence of hitting times $\left\{\sigma_{A}^{n}\right\}_{n \in \mathrm{N}}$ of a set $A(\in \mathcal{E})$ by

$$
\sigma_{A}^{n}:= \begin{cases}0 & \text { if } n=0 \\ \sigma_{A}^{n-1}+\sigma_{A} \circ \theta_{\sigma_{A}^{n-1}} & \text { if } n \geq 1 .\end{cases}
$$

Then $\sigma_{A}^{n}$ means the first time to hit $A$ after time $\sigma_{A}^{n-1}([8])$. We investigate an entry possibility, $P_{\tau_{A}}$, of $A$, and we give a simple and interesting characterization of a possibility, $P_{\sigma_{A}^{n}}$, to hit $A$ first $n$ times.

Proposition 2.1. Let $A \in \mathcal{E}$. Then :

(i) $P_{\tau_{A}} \tilde{s}=G I_{A} \tilde{s} \quad$ for $P$-superharmonic $\tilde{s}$;

(ii) $P_{\sigma_{A}^{n}} \tilde{s}=\left(P G I_{A}\right)^{n} \tilde{s} \quad$ for $P$-superharmonic $\tilde{s}$ and $n \in \mathbf{N}$.

Proof. (i) From Theorem 2.1 and Lemma 2.3(ii) we obtain

$$
P_{\tau_{A}} \tilde{s}=G\left(\tilde{s} \wedge 1_{A}\right)=G I_{A} \tilde{s}
$$

(ii) We prove the equality by induction on $n \in \mathbf{N}$. It is trivial when $n=0$. From (i), $P_{\sigma_{A}} \tilde{s}=P P_{\tau_{A}} \tilde{s}=P G I_{A} \tilde{s}$. So (ii) also holds for $n=1$. Next for every $n \in \mathbf{N},\left(P G I_{A}\right)^{n+1} \tilde{s}$ is $P$-superharmonic since $G I_{A}\left(P G I_{A}\right)^{n} \tilde{s}$ is $P$-superharmonic by Lemma 2.3(ii). Therefore $\left(P G I_{A}\right)^{n} \tilde{s}$ is $P$-superharmonic for all $n \in \mathbf{N}$. Let $n \in \mathbf{N}$. We suppose that (ii) holds for $n$. From (i) and the fact that $\left(P G I_{A}\right)^{n} \tilde{s}$ is $P$-superharmonic,

$$
P_{\sigma_{A}^{n+1}} \tilde{s}=P_{\sigma_{A}} P_{\sigma_{A}^{n}} \tilde{s}=P P_{\tau_{A}}\left(P G I_{A}\right)^{n} \tilde{s}=P G I_{A}\left(P G I_{A}\right)^{n} \tilde{s}=\left(P G I_{A}\right)^{n+1} \tilde{s} .
$$

Thus we obtain (ii) inductively.

\section{3. $\alpha$-recurrent sets}

Definition. Let $\alpha \in(0,1]$. A set $A \in \mathcal{E}(E)$ is called $\alpha$-recurrent provided :

(a) $A$ is non-empty;

(b) $P_{\sigma_{B}^{n}} 1 \geq \alpha$ on $A$ for all $n \in \mathbf{N}$ and all non-empty $B \in \mathcal{E}$ satisfying $B \subset A$.

The $\alpha$-recurrence of a set $A$ means that a possibility to transit infinite times from any point of $A$ to any point of $A$ is greater than $\alpha$.

Lemma 3.1. Let $\beta(\in[0,1])$ be a constant fuzzy set. It holds that

$$
G(\tilde{s} \wedge \beta)=G \tilde{s} \wedge \beta \quad \text { and } \quad P G(\tilde{s} \wedge \beta)=P G \tilde{s} \wedge \beta \quad \text { for } \tilde{s} \in \mathcal{G}(E) .
$$

Especially,

$$
G I_{A}(\beta)=G 1_{A} \wedge \beta \quad \text { and } \quad P G I_{A}(\beta)=P G 1_{A} \wedge \beta \quad \text { for } A \in \mathcal{E}(E) .
$$


Proof. By induction we show

$$
P^{n}(\tilde{s} \wedge \beta)=P^{n} \tilde{s} \wedge \beta \quad \text { for } \tilde{s} \in \mathcal{G}(E) \text { and } n \in \mathbf{N}
$$

First (3.3) holds clearly when $n=0$. Next we have (3.3) for $n=1$ since

$$
P(\tilde{s} \wedge \beta)(x)=\sup _{y \in E}(\tilde{q}(x, y) \wedge \tilde{s}(y) \wedge \beta)=\left(\sup _{y \in E} \tilde{q}(x, y) \wedge \tilde{s}(y)\right) \wedge \beta=P \tilde{s}(x) \wedge \beta \quad x \in E .
$$

Further let $n \in \mathbf{N}$. Assuming that (3.3) holds for $n$, we have

$$
P^{n+1}(\tilde{s} \wedge \beta)=P P^{n}(\tilde{s} \wedge \beta)=P\left(P^{n} \tilde{s} \wedge \beta\right)=P^{n+1} \tilde{s} \wedge \beta
$$

Thus (3.3) holds for all $n \in \mathbf{N}$. Therefore we get (3.1). We also obtain (3.2), taking $\tilde{s}=1_{A}(A \in \mathcal{E}(E))$ in $(3.1)$.

We give simple necessary and sufficient criteria for $\alpha$-recurrence by the transitive closure $P G$.

Proposition 3.1. Let $\alpha \in(0,1]$ and let non-empty $A \in \mathcal{E}(E)$. Then the following statements are equivalent:

(i) $A$ is $\alpha$-recurrent;

(ii) $P G 1_{B} \geq \alpha \wedge 1_{A}$ for non-empty $B \in \mathcal{E}(E)$ satisfying $B \subset A$;

(iii) $P G 1_{\{y\}} \geq \alpha \wedge 1_{A}$ for $y \in A$.

Proof. First we check

$$
\{y\} \in \mathcal{E} \quad \text { for } y \in E .
$$

Let $y \in E$. Then $\{y\} \subset \mathcal{E}(E)$. Put $B_{m}(y):=\{z \in E \mid d(y, z) \geq 1 / m\}$ for $m=1,2, \cdots$, where $d$ denotes a metric on $E$. From [10, Lemma 1.1], $E \backslash\{y\}=\bigcup_{m=1}^{\infty} B_{m}(y) \in \mathcal{E}(E)$. Therefore we obtain (3.4). Next we prove the equivalences of (i) - (iii).

(ii) $\Longrightarrow$ (i) : Let non-empty $B \in \mathcal{E}$ satisfying $B \subset A$. By induction we show

$$
\left(P G I_{B}\right)^{n} 1 \geq \alpha \wedge 1_{A} \quad \text { for } n \in \mathbf{N} .
$$

Inequality (3.5) is trivial for $n=1$. We assume that (3.5) holds for some $n \in \mathbf{N}$. From Lemma 3.1, $\left(P G I_{B}\right)^{n+1} 1=\left(P G I_{B}\right)^{n}\left(P G 1_{B}\right) \geq\left(P G I_{B}\right)^{n}\left(\alpha \wedge 1_{A}\right)=\left(P G I_{B}\right)^{n}(\alpha)=\left(P G I_{B}\right)^{n} \wedge \alpha=\alpha \wedge 1_{A}$.

So (3.5) holds for all $n \in \mathbf{N}$. Therefore we obtain (i), using Proposition 2.1(ii). 
(iii) $\Longrightarrow$ (ii) : Let non-empty $B \in \mathcal{E}(E)$ satisfying $B \subset A$ and let $y \in B$. Then

$$
P G 1_{B} \geq P G 1_{\{y\}} \geq \alpha \wedge 1_{A} .
$$

Therefore we obtain (ii).

(i) $\Longrightarrow$ (iii) : It is trivial from Proposition 2.1(ii).

Thus we complete the proof.

We gives, by the fuzzy relation $\tilde{q}$, a representation of the union of all $\alpha$-recurrent sets.

Theorem 3.1. It holds that

$$
\bigcup_{A \in \mathcal{E}(E): \alpha-\text { recurrent sets }} A=\left\{x \in E \mid \sup _{n \geq 1} \tilde{q}^{n}(x, x) \geq \alpha\right\} \quad \text { for } \alpha \in(0,1] .
$$

Proof. Let $A \in \mathcal{E}(E)$ be $\alpha$-recurrent. From Proposition 3.1, for $x \in A$

$$
P G 1_{\{x\}} \geq \alpha \wedge 1_{A} \geq \alpha \wedge 1_{\{x\}}
$$

Therefore

$$
A \subset\left\{x \in E \mid P G 1_{\{x\}} \geq \alpha \wedge 1_{\{x\}}\right\}=\left\{x \in E \mid \sup _{n \geq 1} \tilde{q}^{n}(x, x) \geq \alpha\right\} .
$$

Conversely let $x \in E$ satisfy $\sup _{n \geq 1} \tilde{q}^{n}(x, x) \geq \alpha$. Then $P G 1_{\{x\}} \geq \alpha \wedge 1_{\{x\}}$. From Proposition 3.1, $\{x\}$ is $\alpha$-recurrent. Therefore

$$
\{x\} \subset \underset{A \in \mathcal{E}(E): \alpha-\text { recurrent sets }}{\bigcup^{\prime}} A .
$$

Thus we obtain this theorem.

\section{The contractive case}

In this section we consider the contractive case in [5] and we give the maximum $\alpha$ recurrent set for the dynamic fuzzy system $X$.

Let $E_{c}$ be a compact subset of $E$. We deal with a dynamic fuzzy system restricted on the compact space $E_{c}$ according to [5]. Let $\mathcal{C}\left(E_{c}\right)$ be the set of all closed subsets of $E_{c}$ and let $\rho$ be the Hausdorff metric on $\mathcal{C}\left(E_{c}\right)$. Let $\mathcal{F}^{0}\left(E_{c}\right)$ be the set of all fuzzy sets $\tilde{s}$ on $E_{c}$ which are upper semi-continuous and satisfy $\sup _{x \in E_{c}} \tilde{s}(x)=1$. Then we note $\mathcal{F}^{0}\left(E_{c}\right) \subset \mathcal{F}\left(E_{c}\right)$. Let $\tilde{p}_{0} \in \mathcal{F}^{0}\left(E_{c}\right)$ be a fuzzy set. Define a sequence of fuzzy sets $\left\{\tilde{p}_{n}\right\}_{n=0}^{\infty}$ by

$$
\tilde{p}_{n+1}(y)=\sup _{x \in E_{c}}\left\{\tilde{p}_{n}(x) \wedge \tilde{q}(x, y)\right\} \quad y \in E_{c} \quad \text { for } n \geq 0 .
$$

The fuzzy set $\tilde{p}_{0}$, in [5], is called an initial fuzzy state and the sequence $\left\{\tilde{p}_{n}\right\}_{n=0}^{\infty}$ is called a sequence of fuzzy states. The fuzzy relation $\tilde{q}$ is also restricted on $E_{c} \times E_{c}$ and it 
is assumed to be continuous on $E_{c} \times E_{c}$ and satisfy $\tilde{q}(x, \cdot) \in \mathcal{F}^{0}(E)$. Define a map $\tilde{r}_{\alpha}: \mathcal{C}\left(E_{c}\right) \rightarrow \mathcal{C}\left(E_{c}\right)(\alpha \in(0,1))$ by

$$
\tilde{r}_{\alpha}(D):= \begin{cases}\{y \mid \tilde{q}(x, y) \geq \alpha \text { for some } x \in D\} & \text { for } \alpha>0, D \in \mathcal{C}\left(E_{c}\right), D \neq \emptyset \\ \operatorname{cl}\{y \mid \tilde{q}(x, y)>0 \text { for some } x \in D\} & \text { for } \alpha=0, D \in \mathcal{C}\left(E_{c}\right), D \neq \emptyset \\ E_{c} & \text { for } 0 \leq \alpha \leq 1, D=\emptyset\end{cases}
$$

In the sequel we assume the following contractive property for the fuzzy relation $\tilde{q}$ (see [5, Section 2]) : There exists a real number $\beta \in(0,1)$ satisfying

$$
\rho\left(\tilde{r}_{\alpha}(A), \tilde{r}_{\alpha}(B)\right) \leq \beta \rho(A, B) \text { for all } A, B \in \mathcal{C}\left(E_{c}\right) \text { and all } \alpha \in(0,1) .
$$

Then we have proved a convergence of the sequence of fuzzy states $\left\{\tilde{p}_{n}\right\}_{n=0}^{\infty}$ defined by (4.1).

Lemma 4.1 ([5, Theorem 1]).

(i) There exists a unique fuzzy state $\tilde{p} \in \mathcal{F}^{0}\left(E_{c}\right)$ satisfying

$$
\tilde{p}(y)=\max _{x \in E_{c}}\{\tilde{p}(x) \wedge \tilde{q}(x, y)\} \quad y \in E_{c} .
$$

(ii) The sequence $\left\{\tilde{p}_{n}\right\}_{n=0}^{\infty}$ converges to a unique solution $\tilde{p} \in \mathcal{F}^{0}\left(E_{c}\right)$ of (4.2) independently of the initial fuzzy state $\tilde{p}_{0}$. Namely,

$$
\lim _{n \rightarrow \infty} \tilde{p}_{n}=\tilde{p}
$$

where the convergence means $\sup _{\alpha \in[0,1]} \rho\left(\tilde{p}_{n, \alpha}, \tilde{p}_{\alpha}\right) \rightarrow 0(n \rightarrow \infty)$ provided $\tilde{p}_{n, \alpha}, \tilde{p}_{\alpha}$ are $\alpha$-cuts $(\alpha \in[0,1])$ for the fuzzy states $\tilde{p}_{n}, \tilde{p}$ respectively.

First we give a solution of (4.2).

Proposition 4.1. The $\alpha$-cut of the solution $\tilde{p}$ of (4.2) is

$$
\tilde{p}_{\alpha}=\mathrm{cl}\left\{x \in E_{c} \mid \sup _{n \geq 1} \tilde{q}^{n}(x, x) \geq \alpha\right\} \quad \text { for } \alpha \in(0,1] .
$$

Proof. First we prove

$$
\sup _{n \geq 1} \tilde{q}^{n}(x, x) \leq \tilde{p}(x) \quad x \in E_{c} .
$$

Let $\alpha \in(0,1]$ and $x \in E_{c}$ satisfy $\sup _{n \geq 1} \tilde{q}^{n}(x, x) \geq \alpha$. For each $\alpha^{\prime}<\alpha$ there exists $n^{\prime} \geq 1$ such that

$$
x \in \tilde{r}_{\alpha^{\prime}}^{n^{\prime}}(\{x\}),
$$


where $\tilde{r}_{\alpha^{\prime}}^{1}:=\tilde{r}_{\alpha^{\prime}}$ and $\tilde{r}_{\alpha^{\prime}}^{n+1}:=\tilde{r}_{\alpha^{\prime}}\left(\tilde{r}_{\alpha^{\prime}}^{n}\right)$ for $n \geq 1$. Then, by induction, we shall check

$$
x \in \tilde{r}_{\alpha^{\prime}}^{n^{\prime} m}(\{x\}) \quad \text { for all } m \geq 1 \text {. }
$$

(4.4) is trivial for $m=1$. We assume that (4.4) holds for $m=1,2, \cdots, l$. From the definition,

$$
x \in \tilde{r}_{\alpha^{\prime}}^{n^{\prime} l}(\{x\}) \subset \bigcup_{y \in \hat{r}_{\alpha^{\prime}}^{n^{\prime}}(\{x\})} \tilde{r}_{\alpha^{\prime}}^{n^{\prime} l}(\{y\})=\tilde{r}_{\alpha^{\prime}}^{n^{\prime}(l+1)}(\{x\}) .
$$

Therefore we obtain (4.4) inductively. On the other hand, considering a case of $\tilde{p}_{0}:=1_{\{z\}}$ $\left(z \in E_{c}\right)$ in (4.1), from Lemma 4.1(ii) and [5, Lemma 1],

$$
\lim _{n \rightarrow \infty} \rho\left(\tilde{r}_{\alpha^{\prime}}^{n}(\{z\}), \tilde{p}_{\alpha^{\prime}}\right)=0 \quad \text { for all } z \in E_{c} .
$$

From (4.4) and (4.5), we obtain $x \in \tilde{p}_{\alpha^{\prime}}$ for $\alpha^{\prime}<\alpha$. Therefore we get $x \in \tilde{p}_{\alpha}$, using Lemma 4.1(i) and [5, Lemma $3(\mathrm{i}, \mathrm{b})]$. Thus we get $(4.3)$.

Let $x \in E_{c}$. Next, considering a case of $\tilde{p}_{0}:=1_{\{x\}}$ in (4.1), we can easily check

$$
\tilde{q}^{n}(x, x)=\tilde{p}_{n}(x) \quad \text { for all } n \geq 1 \text {. }
$$

Together with (4.3), we obtain

$$
\tilde{p}_{m}(x) \leq \sup _{n \geq 1} \tilde{q}^{n}(x, x) \leq \tilde{p}(x) \quad x \in E_{c} \quad \text { for all } m \geq 1 .
$$

By Lemma 4.1(ii), we get

$$
\tilde{p}_{\alpha}=\operatorname{cl}\left\{x \in E_{c} \mid \sup _{n \geq 1} \tilde{q}^{n}(x, x) \geq \alpha\right\} \quad \text { for all } \alpha \in(0,1] .
$$

Therefore the proof is completed.

Finally we prove that the closure of the union of all $\alpha$-recurrent sets equals to $\alpha$-cuts of the limit fuzzy state $\tilde{p}$. Now we compare (1.1) and (4.1). Using the inverse fuzzy relation $\hat{q}([3$, Section 3.2]):

$$
\hat{q}(x, y):=\tilde{q}(y, x) \quad x, y \in E_{c},
$$

we find that (4.1) follows

$$
\tilde{p}_{n+1}(x)=\sup _{x \in E_{c}}\left\{\hat{q}(x, y) \wedge \tilde{p}_{n}(y)\right\} \quad x \in E_{c} \quad \text { for } n \geq 0 .
$$

Therefore we can apply the results in Sections $1-3$ to a dynamic fuzzy system defined by the inverse fuzzy relation $\hat{q}$.

\section{Theorem 4.1.}

$$
\tilde{p}_{\alpha}=\operatorname{cl}\left\{x \in E_{c} \mid \sup _{n \geq 1} \tilde{q}^{n}(x, x) \geq \alpha\right\}=\operatorname{cl}\left(\bigcup_{A \in \mathcal{E}(E): \alpha-\text { recurrent sets }} A\right) \quad \text { for } \alpha \in(0,1] .
$$


Further it is the maximum $\alpha$-recurrent set for $X$.

Proof. From the definition of the inverse fuzzy relation $\hat{q}$, we can easily check

$$
\hat{q}^{n}(x, x)=\tilde{q}^{n}(x, x) \quad x \in E_{c}, n \geq 1
$$

where, in the same way as $\left\{\tilde{q}^{n}\right\}_{n \geq 1}$ of Section 1 , we define

$$
\hat{q}^{1}(x, y):=\hat{q}(x, y) \quad \text { and } \quad \hat{q}^{n+1}(x, y):=\sup _{z \in E_{c}}\left\{\hat{q}^{n}(x, z) \wedge \hat{q}(z, y)\right\} \quad x, y \in E_{c}, n \geq 1 .
$$

From Proposition 4.1,

$$
\tilde{p}_{\alpha}=\mathrm{cl}\left\{x \in E_{c} \mid \sup _{n \geq 1} \hat{q}^{n}(x, x) \geq \alpha\right\}=\mathrm{cl}\left\{x \in E_{c} \mid \sup _{n \geq 1} \hat{q}^{n}(x, x) \geq \alpha\right\} \quad \text { for } \alpha \in(0,1] .
$$

This equality means that the closure of the union of all $\alpha$-recurrent sets for the fuzzy relation $\tilde{q}$ equals to one for the inverse fuzzy relation $\hat{q}$, considering Theorem 3.2 for the dynamic fuzzy systems defined by the fuzzy relations $\tilde{q}$ and $\hat{q}$. Therefore we obtain (4.6). Finally (4.5) means that $\tilde{p}_{\alpha}$ is the maximum $\alpha$-recurrent.

\section{The monotone case}

In general, there does not always exist the maximum $\alpha$-recurrent set for the dynamic fuzzy system $X$, however we can consider the existence of the maximal $\alpha$-recurrent sets. In this section we deal with a case when the transition fuzzy relation $\tilde{q}$ has a certain monotone property (see Section 6 for numerical examples). Then we prove the existence of at most countable arcwise connected maximal $\alpha$-recurrent sets.

In this section we use the notations in Sections $1-3$. Further we introduce the following notations of $\alpha$-cuts ([5, Section 2]) :

$$
\begin{gathered}
\tilde{q}_{\alpha}(x):=\{y \in E \mid \tilde{q}(x, y) \geq \alpha\} \quad \text { for } x \in E \text { and } \alpha \in(0,1] \\
\tilde{q}_{\alpha}(A):=\bigcup_{x \in A} \tilde{q}_{\alpha}(x) \text { for } A \in \mathcal{E}(E) \text { and } \alpha \in(0,1] \\
\tilde{q}_{0}(A):=\operatorname{cl}\left(\bigcup_{\alpha>0} \tilde{q}_{\alpha}(A)\right) \text { for } A \in \mathcal{E}(E) .
\end{gathered}
$$

For $\alpha \in(0,1]$ and $x \in E$ we define a sequence $\left\{\tilde{q}_{\alpha}^{m}(x)\right\}_{m=1,2, \cdots}$ :

$$
\tilde{q}_{\alpha}^{1}(x):=\tilde{q}_{\alpha}(x) ; \quad \text { and } \quad \tilde{q}_{\alpha}^{m+1}(x):=\tilde{q}_{\alpha}\left(\tilde{q}_{\alpha}^{m}(x)\right) \text { for } m=1,2, \cdots .
$$

We also need some elementary notations in the finite dimensional Euclidean space $E$ : $x+y$ denotes the sum of $x, y \in E$ and $\gamma x$ denotes the product of a real number $\gamma$ and $x \in E$. We put $A+B:=\{x+y \mid x \in A, y \in B\}$ for $A, B \in \mathcal{E}(E)$. Then we define a half line on $E$ by

$$
l(x, y):=\{\gamma(y-x) \mid \text { real numbers } \gamma \geq 0\} \quad \text { for } x, y \in E
$$


Definition. We call a transition fuzzy relation $\tilde{q}$ unimodal provided that $\tilde{q}_{\alpha}(x)$ are bounded closed convex subsets of $E$ for all $\alpha \in(0,1]$ and all $x \in E$.

Definition. We call a unimodal transition fuzzy relation $\tilde{q}$ monotone provided that

$$
\tilde{q}_{\alpha}(y) \subset \tilde{q}_{\alpha}(x)+l(x, y) \text { for all } \alpha \in(0,1] \text { and all } x, y \in E \text {. }
$$

From now on we deal with only unimodal fuzzy relations $\tilde{q}$, which is monotone and continuous on $E \times E$. The monotonicity is a natural extension of one-dimensional models with the linear structure in [11] and means that the fuzzy relations $\tilde{q}$ keeps the partial order of fuzzy numbers (see (C.iii') in Section 6).

Lemma 5.1. Assume that $\tilde{q}$ is monotone. Let $\alpha \in(0,1]$. If $x \in E$ satisfies $x \in$ $\bigcup_{m=1}^{\infty} \tilde{q}_{\alpha}^{m}(x)$, then $x \in \tilde{q}_{\alpha}(x)$.

Proof. Let $x(\in E)$ satisfy $x \notin \tilde{q}_{\alpha}(x)$. We put

$$
C_{+}:=\bigcup_{y \in \tilde{q}_{\alpha}(x)}\left\{\tilde{q}_{\alpha}(x)+l(x, y)\right\}
$$

Since $\tilde{q}$ is monotone, we can easily check $C_{+}$is convex and we have

$$
\tilde{q}_{\alpha}^{2}(x)=\bigcup_{y \in \tilde{q}_{\alpha}(x)} \tilde{q}_{\alpha}(y) \subset C_{+}
$$

Here we show

$$
\bigcup_{y \in C_{+}} \tilde{q}_{\alpha}(y) \subset C_{+}
$$

Let $z \in \bigcup_{y \in C_{+}} \tilde{q}_{\alpha}(y)$. Since $\tilde{q}$ is monotone, there exists $y_{1} \in C_{+}$such that $z \in \tilde{q}_{\alpha}(x)+$ $l\left(x, y_{1}\right)$. So there exists $y_{2} \in \tilde{q}_{\alpha}(x)$ such that $y_{1} \in \tilde{q}_{\alpha}(x)+l\left(x, y_{2}\right)$. From the definitions, there exist $z_{1} \in \tilde{q}_{\alpha}(x)$ and a real number $\gamma_{1} \geq 0$ such that

$$
z=z_{1}+\gamma_{1}\left(y_{1}-x\right)
$$

and there exist $z_{2} \in \tilde{q}_{\alpha}(x)$ and a real number $\gamma_{2} \geq 0$ such that

$$
y_{1}=z_{2}+\gamma_{2}\left(y_{2}-x\right) \text {. }
$$

Since $\tilde{q}$ is unimodal, from (5.3) and (5.4) we obtain that

$$
z=z_{1}+\gamma_{1}\left(z_{2}+\gamma_{2}\left(y_{2}-x\right)-x\right)=z_{1}+\left(\gamma_{1}+\gamma_{1} \gamma_{2}\right)\left(\frac{\gamma_{1} z_{2}+\gamma_{1} \gamma_{2} y_{2}}{\gamma_{1}+\gamma_{1} \gamma_{2}}-x\right) \in C_{+} \quad \text { if } \gamma_{1}>0
$$

and that $z=z_{1} \in C_{+}$if $\gamma_{1}=0$. Thus we get (5.2). Therefore from (5.1) and (5.2)

$$
\tilde{q}_{\alpha}^{3}(x)=\bigcup_{y \in \tilde{q}_{\alpha}^{2}(x)} \tilde{q}_{\alpha}(y) \subset \bigcup_{y \in C_{+}} \tilde{q}_{\alpha}(y) \subset C_{+} .
$$


Thus using (5.2) inductively, we obtain

$$
\bigcup_{m=1}^{\infty} \tilde{q}_{\alpha}^{m}(x) \subset C_{+}
$$

On the other hand we show $x \notin C_{+}$. If $x \in C_{+}$, then there exist $z, y \in \tilde{q}_{\alpha}(x)$ and a real number $\gamma \geq 0$ such that $x=z+\gamma(y-x)$. Therefore

$$
x=\frac{z+\gamma y}{1+\gamma} \in \tilde{q}_{\alpha}(x) \text {. }
$$

This contradicts the assumption on $x$ at the beginning of this proof. Therefore we get $x \notin C_{+}$. Together (5.5), this implies

$$
x \notin \bigcup_{m=1}^{\infty} \tilde{q}_{\alpha}^{m}(x)
$$

Thus we obtain this lemma.

When $\tilde{q}$ is monotone, Theorem 3.1 is reduced to the following representation (5.6), which is easy to calculate.

Theorem 5.1. Assume that $\tilde{q}$ is monotone. Let $\alpha \in(0,1]$. Then

$$
\bigcup_{A \in \mathcal{E}(E): \alpha-\text { recurrent sets }} A=\{x \in E \mid \tilde{q}(x, x) \geq \alpha\} .
$$

Proof. Let $x_{1} \in\{x \in E \mid \tilde{q}(x, x) \geq \alpha\}$. Then $P 1_{\left\{x_{1}\right\}}\left(x_{1}\right) \geq \alpha$. So $P G 1_{\left\{x_{1}\right\}} \geq P 1_{\left\{x_{1}\right\}} \geq$ $\alpha 1_{\left\{x_{1}\right\}}$. Therefore $\left\{x_{1}\right\}$ is $\alpha$-recurrent and so we obtain

$$
\{x \in E \mid \tilde{q}(x, x) \geq \alpha\} \subset \bigcup_{A \in \mathcal{E}(E): \alpha-\text { recurrent sets }} A .
$$

Conversely let $A(\in \mathcal{E}(E))$ be $\alpha$-recurrent. Let $x_{1} \in A$. From Proposition 3.1,

$$
P G 1_{\left\{x_{1}\right\}} \geq \alpha 1_{A} \geq \alpha 1_{\left\{x_{1}\right\}} .
$$

Therefore

$$
x_{1} \in \bigcup_{m=1}^{\infty} \hat{q}_{\alpha^{\prime}}^{m}\left(x_{1}\right) \text { for all } \alpha^{\prime}<\alpha .
$$

From Lemma 3.2 we obtain

$$
x_{1} \in \tilde{q}_{\alpha^{\prime}}\left(x_{1}\right) \text { for all } \alpha^{\prime}<\alpha .
$$

Namely we get $\tilde{q}\left(x_{1}, x_{1}\right) \geq \alpha^{\prime}$ for all $\alpha^{\prime}<\alpha$. So we get $\tilde{q}\left(x_{1}, x_{1}\right) \geq \alpha$. Therefore $A \subset\{x \in E \mid \tilde{q}(x, x) \geq \alpha\}$. Thus we establish this theorem.

We need the following assumption on $\tilde{q}$, which is technical but not so strong. It means that the function $\tilde{q}$ does not have flat areas as a curved surface (Section 6). 
Assumption (A). For $\alpha \in(0,1)$,

$$
\operatorname{int}\{(x, y) \in E \times E \mid \tilde{q}(x, y) \geq \alpha\}=\{(x, y) \in E \times E \mid \tilde{q}(x, y)>\alpha\},
$$

where int denotes the interior of a set.

Since $\tilde{q}$ is continuous, $\{x \in E \mid \tilde{q}(x, x) \geq \alpha\}$ is represented by a disjoint sum of at most countable arcwise connected closed sets $([4])$, we represent it by

$$
\{x \in E \mid \tilde{q}(x, x) \geq \alpha\}=\bigcup_{n \in \mathbb{N}(\alpha)} F_{\alpha, n} \quad \text { for } \alpha \in(0,1),
$$

where $F_{\alpha, n}$ are arcwise connected closed subsets of $E$ and we put the index set $\mathbf{N}(\alpha)(\subset \mathbf{N})$.

Lemma 5.2. We suppose Assumption (A). Let $\alpha \in(0,1)$ and $n \in \mathbf{N}(\alpha)$. Then $F_{\alpha, n}$ is $\alpha$-recurrent.

Proof. We write the interior of $F_{\alpha, n}$ by $F_{\alpha, n}^{o}$. First we prove that $F_{\alpha, n}^{o}$ is $\alpha$-recurrent. Let $x_{0} \in F_{\alpha, n}^{o}$. Let $c\left(x_{0}\right)$ be an arc in $F_{\alpha, n}^{o}$, which is connected from $x_{0}$ to a boundary point of $F_{\alpha, n}$. We consider along the arc $c\left(x_{0}\right)$. Then we show

$$
c\left(x_{0}\right) \cap F_{\alpha, n}^{o} \subset \bigcup_{m \geq 1} \tilde{q}_{\alpha}^{m}\left(x_{0}\right) .
$$

Let $x_{1}$ be the first point arriving at the boundary of $\tilde{q}_{\alpha}\left(x_{0}\right)$ along $c\left(x_{0}\right)$. If either there do not exist such points or $x_{1}$ is a boundary point of $F_{\alpha, n}$, then $c\left(x_{0}\right) \subset \tilde{q}_{\alpha}\left(x_{0}\right)$ and clearly (5.7) holds. Therefore it is sufficient to consider a case of $x_{1} \in F_{\alpha, n}^{o}$. Since $x_{0} \in F_{\alpha, n}^{o}$, we have $x_{0} \in\left(\tilde{q}_{\alpha}\left(x_{0}\right)\right)^{\circ}$ and $d\left(x_{0}, x_{1}\right)>0$ from Assumption (A). From $x_{1} \in F_{\alpha, n}^{o} \cap c\left(x_{0}\right)$, we also define $x_{2}$ the first point arriving at the boundary of $\tilde{q}_{\alpha}\left(x_{1}\right)$ along $c\left(x_{0}\right)$. If either there do not exist such points or $x_{2}$ is a boundary point of $F_{\alpha, n}$, then similarly $c\left(x_{0}\right) \subset$ $\tilde{q}_{\alpha}\left(x_{1}\right) \subset \tilde{q}_{\alpha}^{2}\left(x_{0}\right)$ and (5.7) holds. Therefore it is sufficient to consider a case of $x_{2} \in F_{\alpha, n}^{o}$. Thus it is sufficient to check a sequence $\left\{x_{l}\right\}_{l=0,1,2, \ldots}$ which is defined successively in such a manner and which has the following three properties (Fig. 5.1):

(a) $x_{l} \in F_{\alpha, n}^{o} \cap c\left(x_{0}\right)(l=0,1,2, \cdots)$;

(b) $x_{l+1}$ is the boundary point of $\tilde{q}_{\alpha}\left(x_{l}\right)(l=0,1,2, \cdots)$;

(c) $d\left(x_{l}, x_{l+1}\right)>0(l=0,1,2, \cdots)$.

Then there exists a limit point $x=\lim _{l \rightarrow \infty} x_{l}$ since $\tilde{q}_{\alpha}\left(x_{0}\right)$ is bounded and $c\left(x_{0}\right)$ is so. From the property (b) and Assumption (A), $\tilde{q}\left(x_{l}, x_{l+1}\right)=\alpha(l=0,1,2, \cdots)$. Using the continuity of $\tilde{q}$ and Assumption (A), we obtain $\tilde{q}(x, x)=\alpha$ and $x$ is a boundary point of $F_{\alpha, n}$. Therefore (5.7) also holds for this case. Thus we obtain (5.7) in any cases. Since $x_{0}\left(\in F_{\alpha, n}^{o}\right)$ and the $\operatorname{arc} c\left(x_{0}\right)$ are arbitrary in $(5.7)$, we have

$$
F_{\alpha, n}^{o} \subset \bigcup_{m \geq 1} \tilde{q}_{\alpha}^{m}(x) \quad \text { for all } x \in F_{\alpha, n}^{o} \text {. }
$$


This implies that $F_{\alpha, n}^{o}$ is $\alpha$-recurrent for all $\alpha \in(0,1)$ and all $n \in \mathbf{N}(\alpha)$.

Next from the continuity of $\tilde{q}$ and $(5.8)$, for all $\alpha \in(0,1)$ and $x \in F_{\alpha, n}^{o}$ we obtain

$$
F_{\alpha, n}=\bigcap_{\alpha^{\prime}<\alpha} F_{\alpha^{\prime}, n}^{o} \subset \bigcap_{\alpha^{\prime}<\alpha} \bigcup_{m \geq 1} \tilde{q}_{\alpha^{\prime}}^{m}(x)=\left\{y \in E \mid \sup _{m \geq 1} \tilde{q}^{m}(x, y) \geq \alpha\right\} .
$$

Using this result and Proposition 2.1(ii), for $\alpha \in(0,1)$ and $x \in F_{\alpha, n}=\bigcap_{\alpha^{\prime}<\alpha} F_{\alpha^{\prime}, n}^{o}$ we get

$$
\begin{aligned}
F_{\alpha, n} & =\bigcap_{\alpha^{\prime}<\alpha} F_{\alpha^{\prime}, n} \\
& \subset \bigcap_{\alpha^{\prime}<\alpha}\left\{y \in E \mid \sup _{m \geq 1} \tilde{q}^{m}(x, y) \geq \alpha^{\prime}\right\} \\
& =\left\{y \in E \mid \sup _{m \geq 1} \tilde{q}^{m}(x, y) \geq \alpha\right\} \\
& =\left\{y \in E \mid P G I_{\{y\}} 1(x) \geq \alpha\right\} \\
& =\left\{y \in E \mid P_{\sigma_{\{y\}}} 1(x) \geq \alpha\right\} .
\end{aligned}
$$

This means that $F_{\alpha, n}$ is $\alpha$-recurrent.

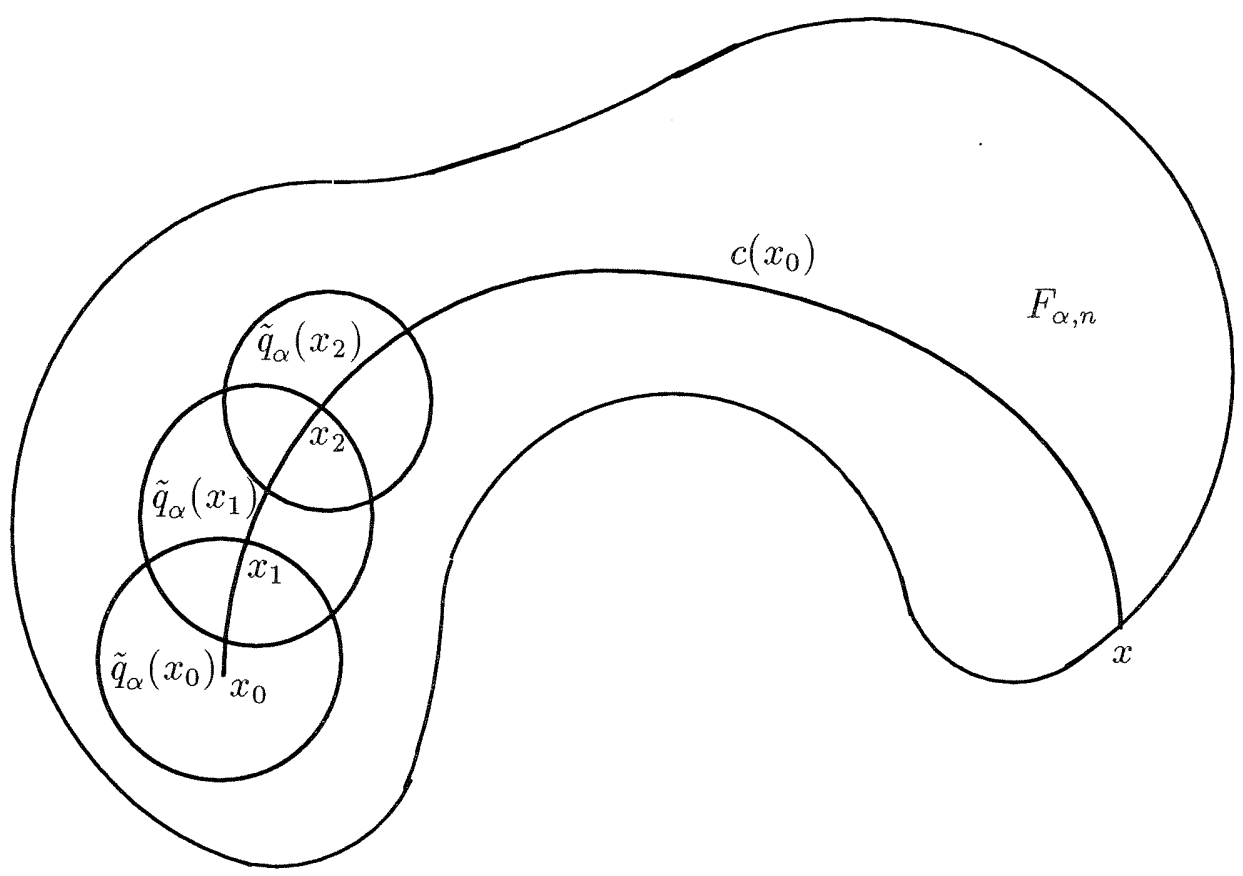

Fig. 5.1. The arcwise connected set $F_{\alpha, n}$ and the sequence $\left\{x_{l}\right\}_{l=0,1,2, \ldots}$

Theorem 5.2. We suppose Assumption (A). Let $\alpha \in(0,1)$. Then maximal $\alpha$-recurrent sets for $X$ are $F_{\alpha, n}(n \in \mathbf{N}(\alpha))$.

Proof. We show that $\alpha$-recurrent sets $F_{\alpha, n}(n \in \mathbf{N}(\alpha))$ are maximal. It is sufficient to prove that $F_{\alpha, n} \cup F_{\alpha, n^{\prime}}$ is not $\alpha$-recurrent, assuming that $\mathbf{N}(\alpha)$ has at least two elements 
$n, n^{\prime}\left(n \neq n^{\prime}\right)$. Now we suppose that there exist $n, n^{\prime} \in \mathbf{N}(\alpha)\left(n \neq n^{\prime}\right)$ such that $F_{\alpha, n} \cup F_{\alpha, n^{\prime}}$ is $\alpha$-recurrent. Then there exist sequences $\left\{x_{m}\right\}_{m=0,1,2, \cdots}$ and $\left\{x_{m}^{\prime}\right\}_{m=0,1,2, \ldots}$ satisfying (a) $-(d)$ :

(a) $x_{0} \in F_{\alpha, n}$ and $\lim _{m \rightarrow \infty} x_{m} \in F_{\alpha, n^{\prime}}$;

(b) $x_{0}^{\prime} \in F_{\alpha, n^{\prime}}$ and $\lim _{m \rightarrow \infty} x_{m}^{\prime} \in F_{\alpha, n}$;

(c) $x_{m+1} \in \tilde{q}_{\alpha}\left(x_{m}\right)(m=0,1,2, \cdots)$;

(d) $x_{m+1}^{\prime} \in \tilde{q}_{\alpha}\left(x_{m}^{\prime}\right)(m=0,1,2, \cdots)$.

We consider the following three cases :

Case when there exists a point $x_{m^{\prime}}$ such that $x_{m^{\prime}} \notin \tilde{q}_{\alpha}\left(x_{m^{\prime}}\right)$ : Then we have

$$
x_{m^{\prime}} \notin\{x \mid \tilde{q}(x, x) \geq \alpha\} .
$$

Since $F_{\alpha, n}$ and $F_{\alpha, n^{\prime}}$ are $\alpha$-recurrent sets, together with (a) - (d), there exists a path from $x_{m^{\prime}}$ to itself through $F_{\alpha, n}$ and $F_{\alpha, n^{\prime}}$, keeping a level of $\tilde{q}$ greater than $\alpha$. Therefore $\left\{x_{m^{\prime}}\right\} \cup F_{\alpha, n} \cup F_{\alpha, n^{\prime}}$ becomes $\alpha$-recurrent. By Theorem 5.1, this fact contradicts (5.9).

Case when there exists a point $x_{m^{\prime}}^{\prime}$ such that $x_{m^{\prime}}^{\prime} \notin \tilde{q}_{\alpha}\left(x_{m^{\prime}}^{\prime}\right)$ : We can derive a contradiction in the same way as the previous case.

Case when $x_{m} \in \tilde{q}_{\alpha}\left(x_{m}\right)(m=0,1,2, \cdots)$ and $x_{m}^{\prime} \in \tilde{q}_{\alpha}\left(x_{m}^{\prime}\right)(m=0,1,2, \cdots)$ : From the assumption that $F_{\alpha, n} \cup F_{\alpha, n^{\prime}}$ is $\alpha$-recurrent, there exists $m^{\prime}$ such that $x_{m^{\prime}} \in F_{\alpha, n}$ and $x_{m^{\prime}+1} \in F_{\alpha, n^{\prime}}$. Therefore

$$
x_{m^{\prime}+1} \in \tilde{q}_{\alpha}\left(x_{m^{\prime}}\right) \cap \tilde{q}_{\alpha}\left(x_{m^{\prime}+1}\right) .
$$

There exists a point $y \notin F_{\alpha, n} \cup F_{\alpha, n^{\prime}}$ such that $y=\lambda x_{m^{\prime}}+(1-\lambda) x_{m^{\prime}+1}(0<\lambda<1)$ since $F_{\alpha, n}$ and $F_{\alpha, n^{\prime}}$ are arcwise connected, closed and disjoint. Then we may take

$$
y \notin\{x \mid \tilde{q}(x, x) \geq \alpha\} .
$$

On the other hand, since $\tilde{q}$ is monotone, we have

$$
\tilde{q}_{\alpha}\left(x_{m^{\prime}+1}\right) \subset \tilde{q}_{\alpha}(y)+l\left(y, x_{m^{\prime}+1}\right)=\tilde{q}_{\alpha}(y)+l\left(x_{m^{\prime}}, x_{m^{\prime}+1}\right)
$$

and

$$
\tilde{q}_{\alpha}\left(x_{m^{\prime}}\right) \subset \tilde{q}_{\alpha}(y)+l\left(y, x_{m^{\prime}}\right)=\tilde{q}_{\alpha}(y)+l\left(x_{m^{\prime}+1}, x_{m^{\prime}}\right) .
$$

From (5.10), (5.12) and (5.13), we obtain

$$
x_{m^{\prime}+1} \in\left(\tilde{q}_{\alpha}(y)+l\left(x_{m^{\prime}}, x_{m^{\prime}+1}\right)\right) \cap\left(\tilde{q}_{\alpha}(y)+l\left(x_{m^{\prime}+1}, x_{m^{\prime}}\right)\right)=\tilde{q}_{\alpha}(y) .
$$


Further since $\tilde{q}_{\alpha}\left(x_{m^{\prime}}\right)$ is convex, from $x_{m^{\prime}} \in \tilde{q}_{\alpha}\left(x_{m^{\prime}}\right)$ and $(5.10)$, we have

$$
y=\lambda x_{m^{\prime}}+(1-\lambda) x_{m^{\prime}+1} \in \tilde{q}_{\alpha}\left(x_{m^{\prime}}\right) .
$$

From (5.14) and (5.15), we get

$$
x_{m^{\prime}+1} \in \tilde{q}_{\alpha}(y) \text { and } y \in \tilde{q}_{\alpha}\left(x_{m^{\prime}}\right) .
$$

Since $F_{\alpha, n}$ and $F_{\alpha, n^{\prime}}$ are $\alpha$-recurrent sets, together with (a) - (d), there exists a path from $y$ to itself through $F_{\alpha, n}$ and $F_{\alpha, n^{\prime}}$, keeping a level of $\tilde{q}$ greater than $\alpha$. By Theorem 5.1, this fact also contradicts (5.11).

Therefore $F_{\alpha, n}(n \in \mathbf{N}(\alpha))$ are maximal $\alpha$-recurrent. By Theorem 5.1, we obtain that maximal $\alpha$-recurrent sets are only $F_{\alpha, n}(n \in \mathbf{N}(\alpha))$.

Remark. When $\alpha=1$, Theorem 5.2 does not hold in general. We consider the following non-contractive numerical example : Let a one-dimensional state space $E=\mathbf{R}$ (the set of all real numbers). We give a fuzzy relation by

$$
\tilde{q}(x, y)=(1-|y-x|) \vee 0, \quad x, y \in \mathbf{R} .
$$

Then we have

$$
\{x \in \mathbf{R} \mid \tilde{q}(x, x)=1\}=\mathbf{R} .
$$

Further we can easily check that every one point set $\{x\} \quad(x \in \mathbf{R})$ are maximal 1-recurrent sets since $\{x\}=\tilde{q}_{1}(x) \quad(x \in \mathbf{R})$.

\section{Numerical examples}

Let a one-dimensional state space $E=\mathbf{R}$. We consider one-dimensional numerical examples. In Section 5 we have assumed the following conditions (C.i) - (C.iv):

(C.i) $\tilde{q}$ is continuous on $E \times E$;

(C.ii) $\tilde{q}$ is unimodal;

(C.iii) $\tilde{q}$ is monotone;

(C.iv) $\tilde{q}$ satisfies Assumption (A).

When $E=\mathbf{R}, \mathcal{F}^{0}(\mathbf{R})$ means all fuzzy numbers on $\mathbf{R}$. From (C.ii), $\tilde{q}_{\alpha}(x)$ are bounded closed intervals of $\mathbf{R}(\alpha \in(0,1], x \in \mathbf{R})$. So we write $\tilde{q}_{\alpha}(x)=\left[\min \tilde{q}_{\alpha}(x), \max \tilde{q}_{\alpha}(x)\right]$, where $\min A(\max A)$ denotes the minimum (maximum resp.) point of a interval $A \subset \mathbf{R}$. Then (C.iii) is equivalent to the following (C.iii') :

(C.iii') $\min \tilde{q}_{\alpha}(\cdot)$ and $\max \tilde{q}_{\alpha}(\cdot)$ are non-decreasing functions on $\mathbf{R}$ for all $\alpha \in(0,1]$.

Next we consider the following partial order $\preceq$ on $\mathcal{F}^{0}(\mathbf{R})($ Nanda $[6])$ : For $\tilde{s}, \tilde{r} \in \mathcal{F}^{0}(\mathbf{R})$, 
$\tilde{s} \preceq \tilde{r}$ means that $\min \tilde{s}_{\alpha} \leq \min \tilde{r}_{\alpha}$ and $\max \tilde{s}_{\alpha} \leq \max \tilde{r}_{\alpha}$ for all $\alpha \in(0,1]$.

Then we can easily find that (C.iii) is equivalent to the following (C.iii") :

(C.iii") If $\tilde{s}, \tilde{r} \in \mathcal{F}^{0}(\mathbf{R})$ satisfy $\tilde{s} \preceq \tilde{r}$, then $Q(\tilde{s}) \preceq Q(\tilde{r})$,

where $Q: \mathcal{F}^{0}(\mathbf{R}) \mapsto \mathcal{F}^{0}(\mathbf{R})$, see (4.1), is defined by

$$
Q \tilde{s}(y)=\max _{x \in \mathbf{R}}\{\tilde{s}(x) \wedge \tilde{q}(x, y)\}, \quad y \in \mathbf{R} \quad \text { for } \tilde{s} \in \mathcal{F}^{0}(\mathbf{R}) .
$$

(C.iii") means that $Q$ preserves the monotonicity on $\mathcal{F}^{0}(\mathbf{R})$ with respect to the order $\preceq$. Finally (C.iv) means that the $\alpha$-level sets $\{x \in \mathbf{R} \mid \tilde{q}(x, x)=\alpha\}(\alpha \in(0,1))$ are drawn by not areas but curved lines. The linear case of [11, Fig. 2] clearly satisfies the above conditions (C.i) - (C.iv), taking the state space $E=(0, \infty)$.

We give an example of monotone fuzzy relations, which is not contractive and does not have the linear structure in [11]. Then we calculate its maximal $\alpha$-recurrent sets.

Example 6.1 (monotone case). We give a fuzzy relation by

$$
\tilde{q}(x, y)=\left(1-\left|y-x^{3}\right|\right) \vee 0, \quad x, y \in \mathbf{R} .
$$

Then $\tilde{q}(x, y)$ satisfies the conditions (C.i) - (C.iv) (see Figure 6.1 for the fuzzy relation $\tilde{q}(x, y)$ and Figure 6.2 for the $\frac{3}{4}$-level sets).

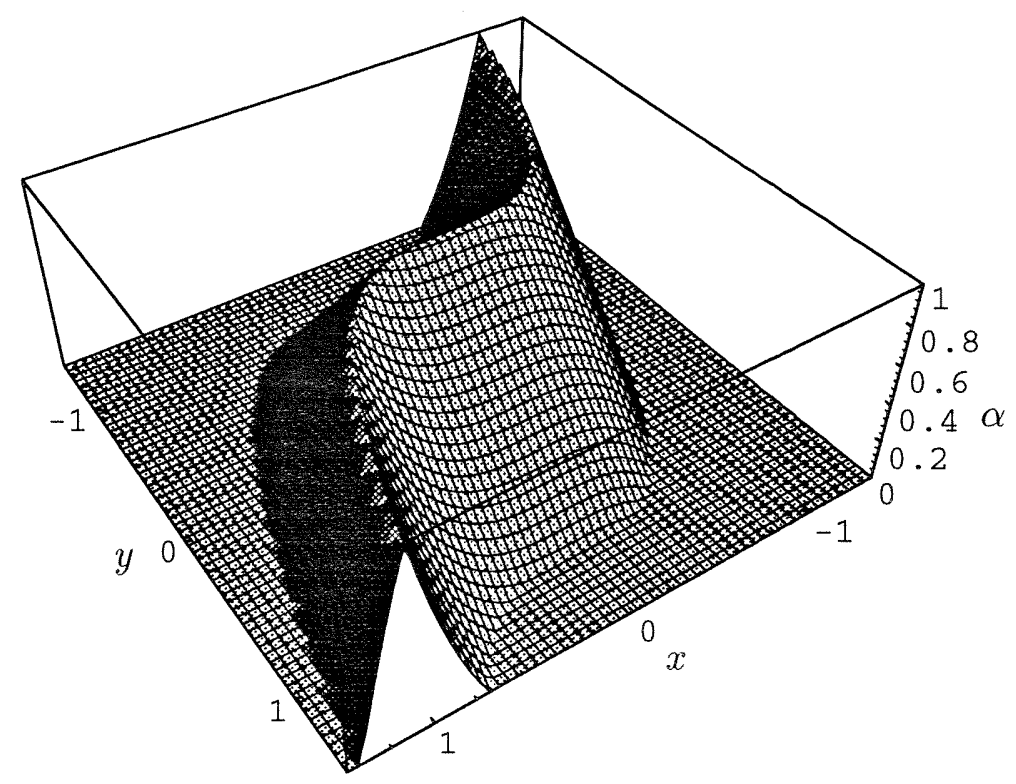

Fig. 6.1 : The monotone fuzzy relation $\tilde{q}(x, y)$. 


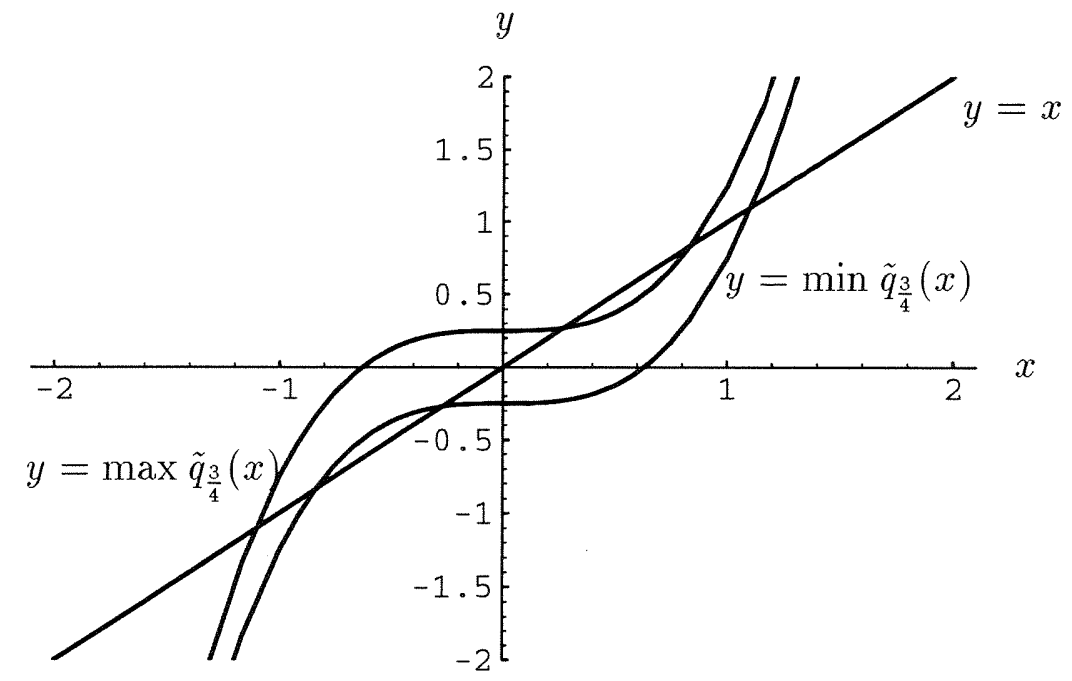

Fig. 6.2. The $\frac{3}{4}$-level sets $\left\{(x, y) \mid \tilde{q}(x, y)=\frac{3}{4}\right\}$.

Then we have

$$
\tilde{q}(x, x)=\left(1-\left|x-x^{3}\right|\right) \vee 0, \quad x \in \mathbf{R} .
$$

Therefore $\mathbf{N}\left(\frac{3}{4}\right)=\{0,1,2\}$ and

$$
\begin{aligned}
& \left\{x \in \mathbf{R} \mid \tilde{q}(x, x) \geq \frac{3}{4}\right\}=F_{\frac{3}{4}, 0} \cup F_{\frac{3}{4}, 1} \cup F_{\frac{3}{4}, 2} \\
& \approx[-1.10716,-0.837565] \cup[-0.269594,0.269594] \cup[0.837565,1.10716] .
\end{aligned}
$$

By Theorem 5.2, the maximal $\frac{3}{4}$-recurrent sets are given by three intervals

$$
\begin{aligned}
& F_{\frac{3}{4}, 0} \approx[-1.10716,-0.837565] \\
& F_{\frac{3}{4}, 1} \approx[-0.269594,0.269594], \\
& F_{\frac{3}{4}, 2} \approx[0.837565,1.10716] .
\end{aligned}
$$

Finally we consider the following numerical example, which is not monotone.

Example 6.2 (non-monotone case). We consider a fuzzy relation

$$
\tilde{q}(x, y)=\max \left\{\left(1-2\left|y-\frac{1}{4} x\right|\right) \vee 0, \min \left\{\left(1-\frac{1}{4}\left|y-\frac{1}{4} x\right|\right) \vee 0, \frac{3}{2}|x| \wedge 1\right\}\right\}, \quad x, y \in \mathbf{R} .
$$

Then $\tilde{q}(x, y)$ satisfies the conditions (C.i), (C.ii) and (C.iv) except for (C.iii) (see Figure 6.3 for the fuzzy relation $\tilde{q}(x, y)$ and Figure 6.4 for the $\frac{3}{4}$-level sets). 


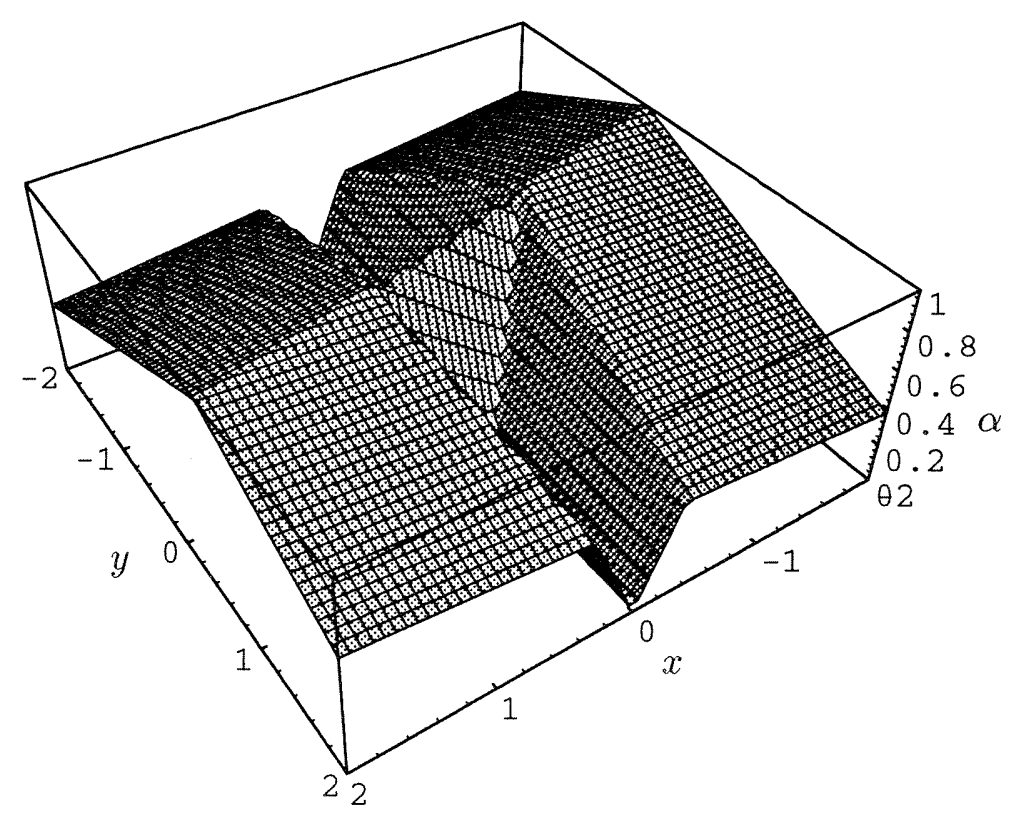

Fig. 6.3 : The non-monotone fuzzy relation $\tilde{q}(x, y)$.

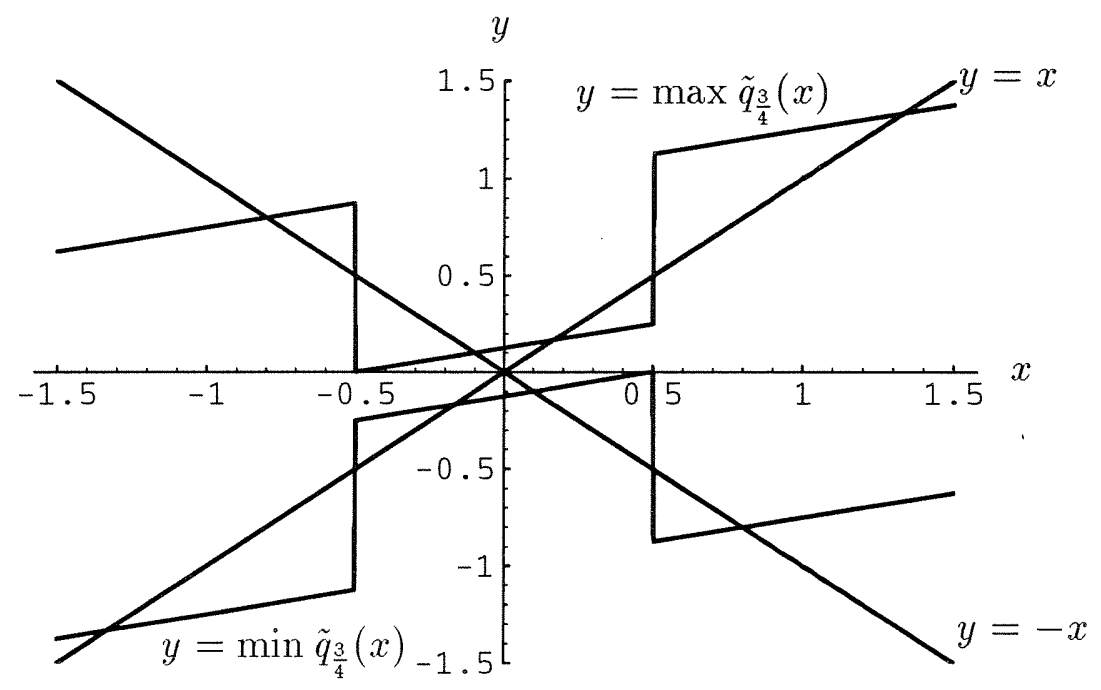

Fig. 6.4. The $\frac{3}{4}$-level set $\left\{(x, y) \mid \tilde{q}(x, y)=\frac{3}{4}\right\}$.

Then

$$
\left\{x \in \mathbf{R} \mid \tilde{q}(x, x) \geq \frac{3}{4}\right\}=\left\{x \in \mathbf{R} \mid \sup _{n \geq 1} \tilde{q}^{n}(x, x) \geq \frac{3}{4}\right\}=\left[-\frac{4}{3},-\frac{1}{2}\right] \cup\left[-\frac{1}{6}, \frac{1}{6}\right] \cup\left[\frac{1}{2}, \frac{4}{3}\right] .
$$

We can easily check the maximal $\frac{3}{4}$-recurrent sets are

$$
\left[-\frac{1}{6}, \frac{1}{6}\right] \text { and }\left[-\frac{4}{3},-\frac{1}{2}\right] \cup\left[\frac{1}{2}, \frac{4}{3}\right] \text {. }
$$


Therefore, in non-monotone case, Theorem 5.2 does not hold in general.

\section{Acknowledgements}

The author is grateful to Prof. M. Kurano for his useful comments and suggestions.

\section{References}

[1] R.E.Bellman and L.A.Zadeh, Decision-making in a fuzzy environment, Management Sci. Ser B. 17 (1970) 141-164.

[2] A.O.Esogbue and R.E.Bellman, Fuzzy dynamic programming and its extensions, TIMS / Studies in Management Sci. 20 (North-Holland, Amsterdam, 1984) 147167.

[3] G.J.Klir and T.A.Folger, Fuzzy Sets, Uncertainty, and Information (Prentice-Hall, London, 1988).

[4] K.Kuratowski, Topology II (Academic Press, New York, 1966).

[5] M.Kurano, M.Yasuda, J.Nakagami and Y.Yoshida, A limit theorem in some dynamic fuzzy systems, Fuzzy Sets and Systems 51 (1992) 83-88.

[6] S.Nanda, On sequences of fuzzy numbers, Fuzzy Sets and Systems 33 (1989) 123-126.

[7] J.Neveu, Discrete-Parameter Martingales (North-Holland, New York, 1975).

[8] D.Revuz, Markov Chains (North-Holland, New York, 1975).

[9] M.Sugeno, Fuzzy measures and fuzzy integral : a survey in M.M.Gupta, G.N.Saridis and B.R.Gaines, Eds., Fuzzy Automata and Decision Processes (North-Holland, Amsterdam, 1977) 89-102.

[10] Y.Yoshida, Markov chains with a transition possibility measure and fuzzy dynamic programming, submitted to Fuzzy Sets and Systems.

[11] Y.Yoshida, M.Yasuda, J.Nakagami and M.Kurano, A potential of fuzzy relations with a linear structure: The contractive case, to appear in Fuzzy Sets and Systems. 